hep-th/0611317

\title{
Cosmological solutions of supercritical string theory
}

\author{
Simeon Hellerman and Ian Swanson \\ School of Natural Sciences, Institute for Advanced Study \\ Princeton, NJ 08540, USA
}

\begin{abstract}
We study quintessence-driven, spatially flat, expanding FRW cosmologies that arise naturally from string theory formulated in a supercritical number of spacetime dimensions. The treelevel potential of the string theory produces an equation of state at the threshold between accelerating and decelerating cosmologies, and the resulting spacetime is globally conformally equivalent to Minkowski space. We demonstrate that exact solutions exist with a condensate of the closed-string tachyon, the simplest of which is a Liouville wall moving at the speed of light. We rely on the existence of this solution to derive constraints on the couplings of the tachyon to the dilaton and metric in the string theory effective action. In particular, we show that the tachyon dependence of the Einstein term must be nontrivial.
\end{abstract}

November 29, 2006 


\section{Introduction}

String theory formulated in a supercritical number of spacetime dimensions provides a natural setting for the study of time-dependent backgrounds in gravitational physics. The simplest classical solutions of supercritical string theory exhibit a string-frame metric that is flat and a dilaton that has a timelike gradient proportional to $\sqrt{D-D_{\text {crit }}}$, where $D_{\text {crit }}$ is the critical number of spacetime dimensions of the theory: 26 for the bosonic string or 10 for the superstring. In this paper we describe these theories in the language of an expanding FRW cosmology driven by quintessence. (Other studies along these lines can be found in [1 9], for example. Recent work also includes [10,11].)

Quintessent cosmologies are defined by an equation of state $w \equiv p / \rho$ taking some fractional negative value not less than -1 . (Situations with $w<-1$ are forbidden by the null energy condition.) In four dimensions, an interesting range of state equations for cosmologists to consider is $-1 \leq w<-1 / 3$ : in this range the scale factor $a(t)$ of the FRW universe accelerates as a function of the proper time $t$ measured by static observers (we refer to this as FRW time). The simplest way to generate models of quintessence is to adopt a matter system described by a single, real scalar field entering the action with a canonical kinetic term and an exponential potential,

$$
\mathcal{V}(\phi)=c \exp (\gamma \phi)
$$

with positive coefficient $c>0$. The value of $\gamma$ in the exponent determines the equation of state $w$ for the theory.

String theory provides several ways of obtaining exponential potentials for scalar fields. The most straightforward is via the tree-level potential for the dilaton that arises in string theories formulated in $D \neq D_{\text {crit }}$ spacetime dimensions. In particular, $c$ is positive for $D>D_{\text {crit }} 1$ The resulting theories are characterized as timelike linear dilaton backgrounds, and they exhibit an extremely simple set of quintessent cosmologies that can be analyzed using worldsheet techniques 2 We should note, however, that the point here is not to suggest that the timelike linear dilaton theory in $D$ dimensions is, by itself, a phenomenologically

\footnotetext{
${ }^{1}$ For $D=D_{\text {crit }}$, the tree-level potential vanishes in the absence of flux or curvature, while for $D<D_{\text {crit }}$, the sign of this potential is negative and the FRW constraint equation cannot be satisfied for a spatially flat universe.

${ }^{2}$ One of the conclusions of [12] was that the absence of well-defined observables at late times can cause trouble for the interpretation of cosmologies characterized by $w<w_{\text {crit }}$. However, backgrounds that asymp-
} 
accurate model of cosmology. We merely intend to demonstrate that string theory can generate simple time-dependent models of rolling massless scalars with flat potentials.

In our next section we study the timelike linear dilaton background in $D$ dimensions from a cosmological perspective: this analysis, in essence, generalizes the presentation in [12] to arbitrary dimension. We demonstrate that the critical equation of state $w_{\text {crit }}$, above which the universe ceases to accelerate, depends on the number of background dimensions $D$. In turn, the global causal structure of the spacetime depends on the value of $w$ relative to $w_{\text {crit }}$. We also introduce the spacetime effective action and analyze the physics of small fluctuations about a quintessent background, showing that the cosmology is stable under perturbations of massless modes. This section is intended to describe many established facts about the supercritical string in a framework that we hope may be useful to cosmologists.

In Section 3 we turn to a worldsheet analysis of the supercritical string. The quintessent cosmological background is unstable against perturbations of a tachyon 3 analogous to the Poincaré-invariant background of the critical string. We demonstrate that, for the supercritical string, there are certain exact solutions for which the tachyon is non-zero. The simplest of these solutions is a 'bubble of nothing' that destroys the spacetime from within, exhibiting behavior similar to that of the Witten solution of [13]. (Other connections between bulk closed-string tachyon condensation and the Witten instanton have been proposed in [14-22].) Relying on worldsheet techniques, we show that these solutions are in fact exact to all orders, and nonperturbatively, in $\alpha^{\prime}$. The existence of such solutions implies constraints on the terms appearing in the effective action, and consistency demands nontrivial tachyon dependence of the Einstein term. In the final section we discuss our conclusions and describe how our work might be extended.

Upon completion of this work, we learned of a paper in preparation 23 that addresses issues related to supercritical string theory. Specifically, the authors of [23] analyze the spacetime physics of the timelike linear dilaton background as a quintessent FRW cosmology, and come to conclusions consistent with our own.

tote to timelike linear dilaton theories exhibit well-defined final states described by a Hilbert space of free particles.

${ }^{3}$ In a generic number of dimensions, tachyon-free timelike linear dilaton theories do not exist. 


\section{Timelike linear dilaton theories as quintessent cos- mology}

In this section we study the timelike linear dilaton background in standard cosmological language. While this section contains no new results, it should serve as a useful review.

Timelike linear dilaton theories are really quintessent cosmologies. This observation is typically obscured by the fact that the string sigma model is naturally described in terms of the string-frame metric rather than the Einstein metric (the two are related by a conformal transformation). The Einstein metric $G_{\mu \nu}^{(E)}$ is, by definition, the metric whose Ricci scalar $R^{(E)}$ appears multiplied by a field-independent coefficient in the action:

$$
\mathcal{L} \sim \frac{1}{2 \kappa^{2}} \sqrt{-\operatorname{det} G^{(E)}} R^{(E)}
$$

(We will use the labels $(S)$ and $(E)$ to denote string and Einstein frame, respectively, and the gravitational coupling $\kappa$ is related to the Newton constant by $G_{N}=\frac{\kappa^{2}}{8 \pi}$.) Alternatively, the

string-frame metric $G_{\mu \nu}^{(S)}$ is the metric whose Ricci scalar $R^{(S)}$ enters the action multiplied by an exponential of the dilaton $\Phi$ :

$$
\mathcal{L} \sim \frac{1}{2 \kappa^{2}} \exp (-2 \Phi) \sqrt{-\operatorname{det} G^{(S)}} R^{(S)}
$$

The precise relation between the two constitutes a Weyl transformation depending on the dilaton:

$$
G_{\mu \nu}^{(S)}=\exp \left(\frac{4 \Phi}{D-2}\right) G_{\mu \nu}^{(E)} .
$$

In this section we aim to study such theories, paying particular attention to the dependence of various cosmological properties on the number of spacetime dimensions. We will restrict attention to cases for which homogeneous spacelike hypersurfaces are flat (in other words, cases for which $k=0$ ). Among other reasons, this allows us to adopt models of the universe that we may assume have undergone significant periods of inflation.

\subsection{Quintessent cosmologies with scalar fields}

We first solve the equations of motion in quintessent cosmological backgrounds in $D$ spacetime dimensions. For this, one may closely follow the treatment in [12]. The metric for a 
spatially flat $(k=0)$ FRW cosmology is

$$
d s^{2}=-d t^{2}+a(t)^{2} d x^{i} d x^{i}
$$

where $i=1, \cdots, D-1$. With the definition $w \equiv p / \rho$, the equation of motion for the scale factor $a(t)$ is

$$
\frac{\ddot{a}}{a}=-\frac{D-3+w(D-1)}{(D-1)(D-2)} \kappa^{2} \rho
$$

(At this stage we are not assuming that $w$ is independent of time.) The constraint equation appears as

$$
H^{2}=\frac{2}{(D-1)(D-2)} \kappa^{2} \rho
$$

where $H$ is the Hubble constant $H \equiv \dot{a} / a$. The matter content of the theory is taken to be a real scalar field $\phi$ with Lagrangian

$$
\mathcal{L}_{\phi}=\frac{1}{\kappa^{2}} \sqrt{-\operatorname{det} G^{(E)}}\left[-\frac{1}{2}\left(\partial_{\mu} \phi\right)\left(\partial^{\mu} \phi\right)-\mathcal{V}(\phi)\right],
$$

where

$$
\mathcal{V}(\phi) \equiv c \exp (\gamma \phi), \quad c, \gamma>0
$$

This yields the following stress tensor (respectively, the energy density and pressure):

$$
\begin{aligned}
& \rho \equiv \frac{1}{\kappa^{2}}\left(\frac{1}{2} \dot{\phi}^{2}+\mathcal{V}\right) \\
& p=\frac{1}{\kappa^{2}}\left(\frac{1}{2} \dot{\phi}^{2}-\mathcal{V}\right) .
\end{aligned}
$$

With this input, the constraint equation becomes

$$
\frac{\dot{\phi}^{2}}{2}+\mathcal{V}=\frac{1}{2}(D-2)(D-1) H^{2}
$$

We assume here that the Einstein term is to remain canonical

$$
\mathcal{L}_{\text {Einstein }}=\frac{1}{2 \kappa^{2}} \sqrt{-\operatorname{det} G^{(E)}} R^{(E)},
$$


and, if the scalar field respects the $(D-1)$-dimensional Poincaré symmetries of the $k=0$ spatial slices, the equation of motion for $\phi$ becomes

$$
\ddot{\phi}+(D-1) H \dot{\phi}=-\mathcal{V}^{\prime}(\phi)
$$

At this point, we adopt the ansatz that our solution exhibits a constant equation of state $w$. It follows that $\dot{\phi}^{2}, H^{2}$ and $\mathcal{V}$ all scale as $t^{-2}$, so we find the general expressions

$$
\begin{aligned}
& \phi(t)=\lambda \log \left(t / t_{1}\right), \\
& a(t)=a_{0}\left(\frac{t}{t_{0}}\right)^{\alpha},
\end{aligned}
$$

for some $\alpha, \lambda$. From the fact that $\mathcal{V}$ scales as $c\left(t_{1} / t\right)^{2}$, we conclude that

$$
\lambda \gamma=-2
$$

Subjecting $\mathcal{V}$ (as a function of time) to the restriction of constant equation of state yields

$$
c t_{1}^{2}=\frac{2}{\gamma^{2}} \frac{1-w}{1+w} .
$$

It follows that the constraint equation implies

$$
\frac{1}{2}(D-1)(D-2) \alpha^{2}=c t_{1}^{2}+\frac{2}{\gamma^{2}} \text {. }
$$

To solve for $\alpha$, we differentiate Eqn. (2.9) with respect to time and use Eqns. (2.13) and (2.10) to determine

$$
\dot{\rho}+\frac{\dot{a}}{a}(D-1)(1+w) \rho=0
$$

This can be integrated to yield

$$
\rho=\rho_{0}\left(\frac{a_{0}}{a}\right)^{(D-1)(1+w)}
$$

To fix $\rho_{0}$, we evaluate the constraint equation at $t_{0}$ and require that $\rho=\rho_{0}$ at time $t_{0}$, which gives

$$
\rho_{0}=\frac{4}{(D-1) t_{0}^{2}(1+w)^{2} \kappa^{2}}
$$


Finally, we substitute Eqn. (2.19) into the constraint equation. This amounts to a direct relation between $\dot{a}$ and $a$, which can again be integrated to yield the following:

$$
\begin{aligned}
\alpha & \equiv \frac{2}{(1+w)(D-1)}, \\
\gamma^{2} & =\frac{2(D-1)(w+1)}{D-2}, \\
w & =-1+\frac{(D-2) \gamma^{2}}{2(D-1)} .
\end{aligned}
$$

Because $c>0$, the energy density $\rho$ is positive-definite, and the cosmological scale $a$ accelerates as a function of FRW time if (and only if) $-1 \leq w<w_{\text {crit }}$, where

$$
w_{\text {crit }}=-\frac{D-3}{D-1}
$$

in $D$ spacetime dimensions.

The global causal structure of the solution depends on whether $w$ is less than, greater than, or equal to the critical value $w_{\text {crit }}$. The spatial slice $t=0$ defines a singularity in all three cases. The precise nature of this singularity and the nature of the asymptotic future $t \rightarrow+\infty$, however, depend on the state equation of the cosmology.

To investigate these issues in greater detail, we begin by demonstrating the coordinate transformation that puts the metric in canonical form for a conformally flat spacetime. In FRW coordinates, we have

$$
d s^{2}=-d t^{2}+a_{0}^{2}\left(\frac{t}{t_{0}}\right)^{4 /(1+w)(D-1)} d x^{i} d x^{i}
$$

Working by analogy from [12], we define a new time coordinate $\bar{t}$ via the equation

$$
\bar{t} \equiv\left(\frac{(D-1)(1+w)}{(D-1) w+(D-3)} t_{0}^{\frac{2}{(D-1)(1+w)}} a_{0}^{-1}\right) t^{\frac{(D-1) w+(D-3)}{(D-1)(1+w)}} .
$$

In these coordinates, the metric takes the form

$$
d s^{2}=\omega(\bar{t})^{2}\left[-d \bar{t}^{2}+d x^{i} d x^{i}\right]=\omega(\bar{t})^{2}\left[-d \bar{t}^{2}+d r^{2}+r^{D-2} d \Omega_{D-2}^{2}\right],
$$

where we have defined

$$
\begin{aligned}
\omega(\bar{t}) & \equiv l\left(\frac{(D-1) w+(D-3)}{(D-1)(1+w)} \bar{t}\right)^{\frac{2}{(D-1) w+(D-3)}}, \\
l & \equiv a_{0}\left(\frac{a_{0}}{t_{0}}\right)^{\frac{2}{(D-1) w+(D-3)}} .
\end{aligned}
$$


In an accelerating universe, the combination

$$
\frac{(D-1)(1+w)}{(D-1) w+(D-3)}
$$

is negative, and the range of $\bar{t}$ is $\bar{t} \in(-\infty, 0)$. The initial singularity occurs at $\bar{t}=-\infty$, and the infinite future lies at $\bar{t}=0$. Alternatively, the combination above is positive for a decelerating cosmology, and $\bar{t}$ spans the range $\bar{t} \in(0, \infty)$. In this latter case the initial singularity occurs at $\bar{t}=0$, and the infinite future is located at $\bar{t}=+\infty$.

For the sake of completeness, we will briefly summarize the structures of the Penrose diagrams appearing for the various ranges of $w$ described above. To construct these diagrams, one ignores the $(D-2)$-sphere fibered over each diagram and conformally compactifies the $(r, t)$ plane using the transformation

$$
r \equiv \frac{\sin \chi}{\cos \chi+\cos \tau}, \quad \bar{t} \equiv \frac{\sin \tau}{\cos \chi+\cos \tau}
$$

In these coordinates, the metric on the $(r, t)$ plane becomes

$$
d s^{2}=\frac{l^{2}}{4} \frac{(\sin |\tau|)^{2 \Delta}}{\left[\cos \left(\frac{\chi+\tau}{2}\right) \cos \left(\frac{\chi-\tau}{2}\right)\right]^{2+2 \Delta}}\left|\frac{(D-1) w+(D-3)}{2(D-1)(1+w)}\right|^{2 \Delta}\left(-d \tau^{2}+d \chi^{2}\right),
$$

with

$$
\Delta \equiv \frac{2}{(D-1) w+(D-3)}
$$

For $-1<w<w_{\text {crit }}$, the constant $\Delta$ is negative and the range of the $\tau$ and $\chi$ coordinates is

$$
\tau \in[-\pi, 0], \quad \chi \in[0, \tau+\pi], \quad \text { (accelerating universe). }
$$

For $w>w_{\text {crit }}$, the quantity $\Delta$ becomes positive and we have

$$
\tau \in[0, \pi], \quad \chi \in[0, \pi-\tau], \quad \text { (decelerating universe). }
$$

In the decelerating case, the state equation lies in the range $w>w_{\text {crit }}$, and it is easy to see that the spatial slice $t=0$ is an ordinary, spacelike Big-Bang singularity with divergent scalar curvature (for $w \neq-1$ ). In addition, future infinity is null, as it is in ordinary Minkowski space. This structure is depicted in detail in Fig. 1.

In the accelerating case $\left(-1<w<w_{\text {crit }}\right)$, the singular hypersurface at $t=0$ is null, in the sense that it is diagonal on a Penrose diagram. Future infinity is spacelike in this range, 


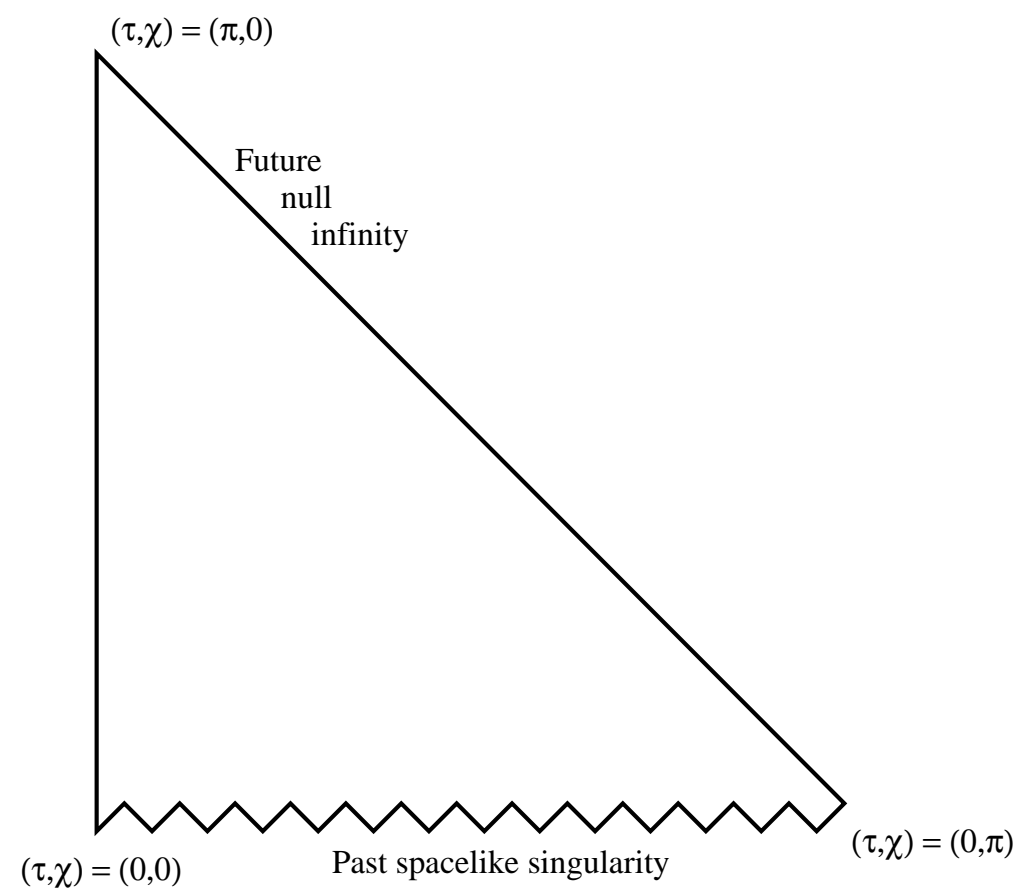

Figure 1: Penrose diagram of the decelerating universe $\left(w>w_{\text {crit }}\right)$. The initial singularity is spacelike, and the future boundary is null.

and all but one point of the future boundary of the Penrose diagram is obscured from the view of any observer by a horizon [12. In particular, a horizon exists at a proper distance

$$
L_{H}=t \frac{(D-1)(1+w)}{|(D-1) w+(D-3)|} .
$$

This horizon recedes at a fixed proper speed, which increases to the speed of light as $w$ approaches $w_{\text {crit }}$ from below. This is depicted in Fig. 2, (There is no horizon for $w \geq w_{\text {crit }}$.)

The liminal case $w=w_{\text {crit }}$ is a hybrid of the two, with a null initial singularity and a null future infinity; the Penrose diagram for this configuration is depicted in Fig. 3. In fact, the liminal quintessent solution is globally conformally equivalent to Minkowski space, and we will find a simple way to understand this fact in our stringy models.

\subsection{Spacetime effective action for supercritical string theory}

We now introduce the spacetime effective action for string theory formulated in a supercritical number of spacetime dimensions. Although we restrict our attention to the bosonic 


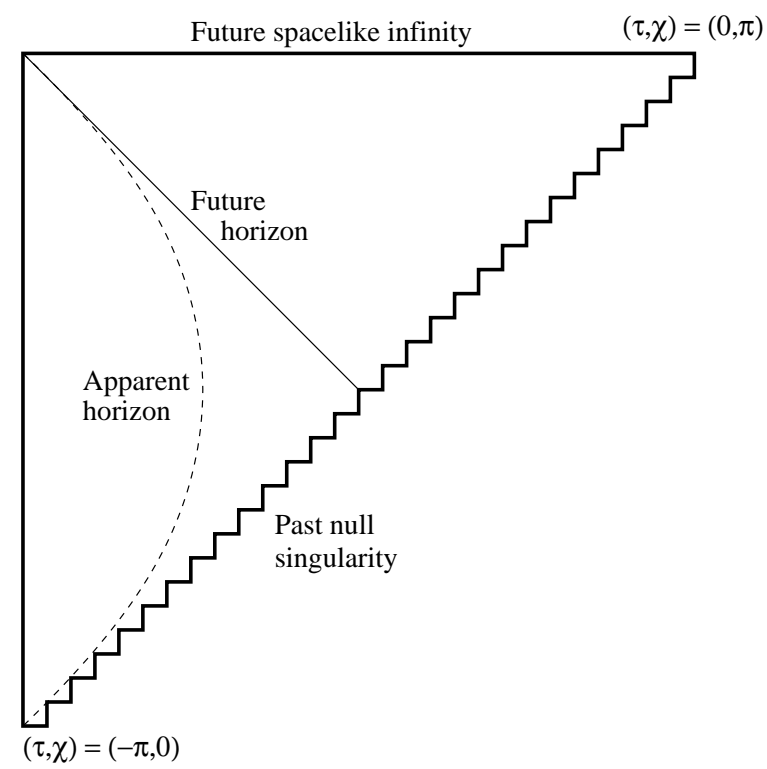

Figure 2: Penrose diagram of the accelerating $\left(-1<w<w_{\text {crit }}\right)$ universe. The initial singularity is null, and the future spacelike boundary is obscured from observers by a horizon.

string, most of the discussion applies to the various closed superstring theories as well. The exact effective action of any string theory is typically a complicated expression involving nonrenormalizable interactions of arbitrarily high dimension. Much of the qualitative physics of string theory, however, is captured by the dynamics of the effective action for light fields, truncated to terms with few derivatives.

For the bosonic string in $D>26$, the effective action for the metric and dilaton appears as

$$
S_{\mathrm{eff}}=\frac{1}{2 \kappa^{2}} \int d^{D} x \sqrt{-\operatorname{det} G^{(S)}} \exp (-2 \Phi)\left[-\frac{D-26}{3 \alpha^{\prime}}+R^{(S)}+4(\partial \Phi)^{2}\right] .
$$

Higher dimension terms are dropped: such terms in the tree-level action are suppressed by powers of $\alpha^{\prime}=1 /\left(2 \pi T_{\text {string }}\right)$, where $T_{\text {string }}$ is the fundamental string tension. Note that the action in Eqn. (2.33) is written in terms of the string-frame metric $G_{\mu \nu}^{(S)}$. We may rewrite the action in terms of the Einstein metric using the field redefinition in Eqn. (2.3). Using the rescaling $\Phi \rightarrow \frac{1}{2} \sqrt{D-2} \phi$, the scalar field appears canonically, and we obtain the following form:

$$
S_{\mathrm{eff}}=\frac{1}{2 \kappa^{2}} \int d^{D} X \sqrt{-\operatorname{det} G^{(E)}}\left[-\frac{2(D-26)}{3 \alpha^{\prime}} \exp \left(\frac{2 \phi}{\sqrt{D-2}}\right)+R^{(E)}-(\partial \phi)^{2}\right] .
$$




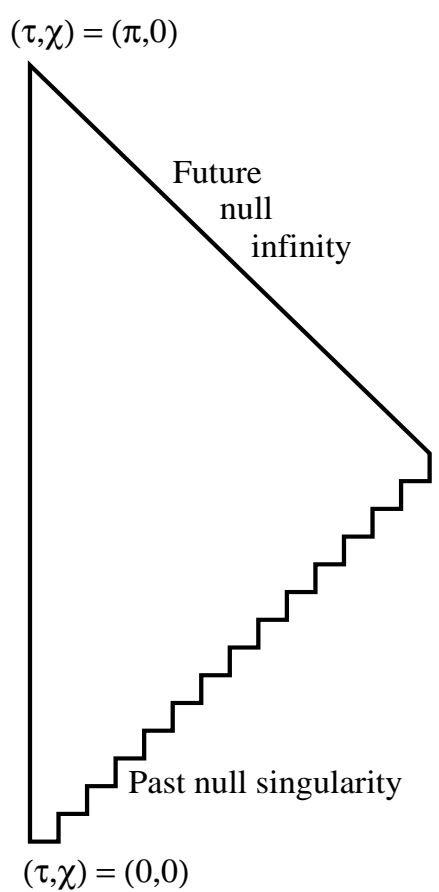

Figure 3: Penrose diagram of the universe with critical equation of state $\left(w=w_{\text {crit }}\right)$. The initial singularity is null, as is the future boundary.

We note that this is the action for a quintessent cosmology, taking the same form as Eqns. (2.7, 2.12) above, with coefficients now defined by the following values:

$$
\gamma=\frac{2}{\sqrt{D-2}}, \quad c=\frac{D-26}{3 \alpha^{\prime}} .
$$

We therefore recover a quintessent solution with equation of state given by the formula in Eqn. (2.21):

$$
w=-\frac{D-3}{D-1}
$$

The tree-level potential of the string theory thus gives rise to an equation of state at the boundary between accelerating and decelerating cosmological backgrounds. As we have seen above, the resulting spacetime is globally conformally equivalent to Minkowski space.

In retrospect, it is inevitable that this should be the case. The worldsheet theory of the string in the timelike linear dilaton background is defined to have a target space with string frame metric $\eta_{\mu \nu}$, and coordinates $X^{\mu}$ that are infinite in extent. A spacetime potential that encodes the worldsheet physics consistently must therefore produce a solution for which the 
metric has those features. The equation of state $w=-\frac{D-3}{D-1}$ is the unique value allowing such a solution.

One important point is that $w$ is negative for any $D \geq 4$. The matter and radiation equations of state are always $w_{\text {mat }}=0$ and $w_{\text {rad }}=+1 /(D-1)$, respectively. Both components have nonnegative $w$, but the component of the stress tensor with the most negative value of $w$ always dominates the evolution of the universe at large scale factor. This means that particle production at early times never changes the late time behavior of the solution.

The general quintessent FRW solution to this system is

$$
\begin{aligned}
\phi & =\phi_{0}-\sqrt{D-2} \ln \left(\frac{t}{t_{0}}\right), \\
a & =\frac{a_{0}}{t_{0}} t .
\end{aligned}
$$

In terms of the original dilaton field $\Phi$, this solution takes the following form:

$$
\Phi=\Phi_{0}-\frac{D-2}{2} \ln \left(\frac{t}{t_{0}}\right)
$$

Since the background metric is conformally flat, it is advantageous to convert to a more natural time coordinate $t_{\text {conf }}$ given by

$$
\begin{aligned}
& t_{\mathrm{FRW}}=t_{0} \exp \left(+\frac{2 q t_{\mathrm{conf}}}{D-2}\right), \\
& t_{\mathrm{conf}}=+\frac{2(D-2)}{q} \ln \left(\frac{t_{\mathrm{FRW}}}{t_{0}}\right),
\end{aligned}
$$

where, for convenience, we have defined a quantity $q$ according to

$$
q \equiv \sqrt{\frac{c}{2}}=\sqrt{\frac{(D-26)}{6 \alpha^{\prime}}} .
$$

In these variables, the Einstein metric is manifestly conformally flat and the dilaton is linear:

$$
\begin{aligned}
d s^{2} & =\frac{a_{0}^{2}}{t_{0}^{2}} t^{2}\left(-d t_{\mathrm{conf}}^{2}+d x^{i} d x^{i}\right)=a^{2} \eta_{\mu \nu} d x^{\mu} d x^{\nu} \\
\Phi & =\Phi_{0}-q t_{\mathrm{conf}}
\end{aligned}
$$

with $x^{0} \equiv t_{\text {conf }}$. 
At this point we can set $a_{0}$ and $t_{0}$ at our convenience. It turns out that a useful choice is for these quantities to be such that the string-frame metric is unit-normalized. Applying the Weyl transformation in Eqn. (2.3), the string-frame metric takes the general form

$$
G_{\mu \nu}^{(S)}=a_{0}^{2} \exp \left(\frac{4 \Phi_{0}}{D-2}\right) \eta_{\mu \nu}
$$

The condition $G_{\mu \nu}^{(S)}=\eta_{\mu \nu}$ therefore implies the following value for $a_{0}$ :

$$
a_{0}=\exp \left(-\frac{2 \Phi_{0}}{D-2}\right)
$$

In turn, by demanding that $a^{2} d t_{\mathrm{conf}}^{2}=d t_{\mathrm{FRW}}^{2}$, we find

$$
a^{2}=\frac{4 q^{2}}{(D-2)^{2}} t_{\mathrm{FRW}}^{2}
$$

From the second line in Eqn. (2.37) $\left(a=a_{0} t / t_{0}\right)$, this translates into the following value for $t_{0}$ :

$$
t_{0}=\frac{(D-2) a_{0}}{\sqrt{2 c}}
$$

In conformal coordinates, the string-frame metric is trivial, and the dilaton is linear in the timelike direction, with gradient

$$
\partial_{x^{0}} \Phi=-q \equiv-\sqrt{\frac{(D-26)}{6 \alpha^{\prime}}} .
$$

This agrees with the known formula for the strength of the dilaton gradient in noncritical bosonic string theory (see, e.g., [24]).

\subsection{Fluctuations of the metric and dilaton}

In time-dependent backgrounds there is no obvious and natural definition of stability. One definition would be to rescale modes in such a way that the kinetic terms are described by a trivial, canonically normalized kinetic term. One may then define an unstable mode as one that grows exponentially in time (once it has been rescaled to be canonical). According to this definition, we will see that there indeed exist 'unstable' modes of the metric and dilaton in timelike linear dilaton theories [25]. 
However, the criterion of stability in terms of the growth of canonical modes is not completely satisfactory. According to this criterion, a spacetime-independent shift of a massless scalar (such as the dilaton) is counted as an unstable mode. Furthermore, various modes of the theory that are pure gauge would also be termed unstable, such as the mode that implements an overall rescaling of the metric, or the longitudinal modes of the NS/NS B-field.

By taking into account the coupling of the background fields to modes of the string, we can formulate a definition of stability that avoids these difficulties [26]. The coupling of a background field to the string is suppressed by a factor of $g_{s}=\exp (\Phi)$. If an exponentially growing mode grows more slowly than $g_{s}^{-1}$, its effect on the remaining degrees of freedom in the theory therefore decreases in time. We may thus define an unstable (stable) mode as one that grows faster (slower) than $g_{s}^{-1}$ at late times. This indeed alleviates the problems noted above: we can see that longitudinal modes of gauge fields have

$$
\text { (amplitude) } / g_{s} \sim \text { const. }
$$

at $t \rightarrow+\infty$, and the same holds for the mode representing a constant shift of the dilaton $\Phi \rightarrow \Phi+$ const.

To illustrate this point more explicitly, let us work out the equations of motion for a massless scalar $\sigma$ coupled with unit strength to the fundamental string:

$$
\mathcal{L}_{\sigma}=-\frac{1}{2 \kappa^{2}} \sqrt{-\operatorname{det} G^{(S)}} e^{-2 \Phi}(\partial \sigma)^{2}=-\frac{1}{2 \kappa^{2}} \sqrt{-\operatorname{det} G^{(E)}}(\partial \sigma)^{2} .
$$

The scalar field has solutions of the form

$$
\sigma=\sigma_{\infty}-\xi t^{-(D-2)}
$$

where $\sigma_{\infty}$ and $\xi$ are constants of motion that can take arbitrary real values. Eqn. (2.50) states that the modes of the field $\sigma$ asymptote to the constant value $\sigma_{\infty}$ as $t \rightarrow \infty$. From the point of view of the Einstein frame, this effect is due to Hubble friction; in the string frame this behavior is understood to be caused by the drag force arising from the interaction between $\sigma$ and the linear dilaton.

As it stands, $\sigma$ is coupled to an Einstein metric that is conformally flat, albeit with a nontrivial conformal factor. A state of the string is necessarily coupled to the flat metric, as the kinetic term of the string sigma model is the flat metric $G^{(S)}$. To recover field fluctuations whose quanta represent normalizable string states, we must introduce a rescaled field $\tilde{\sigma}$ :

$$
\tilde{\sigma} \equiv e^{-\Phi} \sigma
$$


This induces a mass term for the rescaled field that couples to a trivial metric and represents a proper quantum of string. Schematically, we have

$$
e^{-2 \Phi}(\partial \sigma)^{2}=(\partial \tilde{\sigma})^{2}+\tilde{\sigma}^{2}(\partial \Phi)^{2}+2 \tilde{\sigma}(\partial \tilde{\sigma})\left[(\partial \Phi)_{\text {background }}+(\partial \Phi)_{\text {fluctuation }}\right] .
$$

The fluctuation term represents a trilinear vertex that we discard from the outset. The background term is constant, so its dot product with $\tilde{\sigma} \partial \tilde{\sigma}$ amounts to a total derivative. The mass term for the rescaled field is tachyonic and proportional to $-q^{2}$, and the quadratic action for $\tilde{\sigma}$ thus reads

$$
\mathcal{L}_{\tilde{\sigma}} \sim-\frac{1}{2 \kappa^{2}}\left[(\partial \tilde{\sigma})^{2}-q^{2} \tilde{\sigma}^{2}\right]
$$

The criterion for stability we proposed was that $g_{s}$ times the canonical field $\tilde{\sigma}$ not increase exponentially with time. Since $g_{s} \tilde{\sigma}$ is just $\sigma$, the original field appearing in the spacetime action in front of $\exp (-2 \Phi)$, the requirement for physical stability is that modes normalized to have the factor $\exp (-2 \Phi)$ in their kinetic term should shrink exponentially in the future or, at most, remain constant in magnitude.

At this stage, a simple exercise is to find linearized modes of $\tilde{\sigma}$ and $\sigma$ that are plane waves in the spatial directions:

$$
\tilde{\sigma}=\mathcal{A} \sin (\vec{k} \cdot \vec{x}) e^{ \pm \tilde{\Gamma} t_{\text {conf }}}
$$

or

$$
\tilde{\sigma}=\mathcal{A} \sin (\vec{k} \cdot \vec{x}) e^{i \omega t_{\mathrm{conf}}}
$$

where

$$
\tilde{\Gamma}^{2}=q^{2}-\vec{k}^{2}, \quad \omega^{2}=\vec{k}^{2}-q^{2},
$$

and $\mathcal{A}$ is an arbitrary mode amplitude. Moving back to FRW time and the $\sigma$ normalization for the scalar field, we recover the form

$$
\sigma=e^{\Phi_{0}} \mathcal{A} \sin (\vec{k} \cdot \vec{x})\left(\frac{t}{t_{0}}\right)^{\mathcal{B}_{ \pm}},
$$

for the overdamped modes, 4 where

$$
\begin{aligned}
\mathcal{B}_{ \pm} & \equiv \frac{(D-2)}{2 q} \Gamma_{ \pm} \\
\Gamma_{ \pm} & \equiv \pm \sqrt{q^{2}-\vec{k}^{2}}-q .
\end{aligned}
$$

\footnotetext{
${ }^{4}$ These modes are referred to as "pseudotachyons" in [23].
} 
At $k=0$ and $\Gamma_{+}=0$, a background value of the mode represents a "condensation" of the massless field, which shifts the asymptotic value $\sigma_{\infty}$. At the $k=0, \Gamma_{-}=-2 q$ end, we have

$$
\sigma=e^{\Phi_{0}}\left(\frac{t}{t_{0}}\right)^{-(D-2)} .
$$

As such, the underdamped modes of the massless field are given by

$$
\sigma=\exp \left(\Phi_{0}\right) \mathcal{A} \sin \vec{k} \cdot \vec{x}\left(\frac{t}{t_{0}}\right)^{-\frac{D-2}{2}}\left(\frac{t}{t_{0}}\right)^{ \pm \frac{i(D-2) \omega}{2 q}} .
$$

It is clear that these are all stable, in the sense that they asymptote to zero at late times.

Upon adding a mass term of the form

$$
-\frac{1}{2} \exp (-2 \Phi) \sqrt{-\operatorname{det} G^{(S)}} m^{2} \sigma^{2}
$$

to the Lagrangian, we find that the system again breaks into a set of overdamped modes with $\vec{k}^{2}<q^{2}-m^{2}$, and underdamped modes with $\vec{k}^{2}>q^{2}+m^{2}$. Under the replacement $\vec{k}^{2} \rightarrow \vec{k}^{2}+m^{2}$, the form of these modes is the same as those in Eqns. (2.54- 2.58), and all $\sigma$-modes decay exponentially in time when $m^{2} \geq 0$.

When a mode acquires a background value obeying the equations of motion, we may ask what the resulting effect is on string propagation as $t \rightarrow \infty$. The specific effect from the background is to add a term

$$
\mathcal{O} \exp (\Phi(t)) \tilde{\sigma}(x, t)=\sigma(x, t)
$$

to the string worldsheet action, where $\mathcal{O}$ is some operator in the $2 \mathrm{D}$ conformal field theory describing the dynamics of a string. In all cases, $\sigma$ decreases exponentially or stays constant as long as $m^{2} \geq 0$. The effect of the mode on string propagation decreases exponentially with time, or at most stays constant as $t \rightarrow \infty$. It is therefore best to not think of these modes as describing physical instabilities of any kind, despite the fact that the quantum mechanics problem for the canonical mode $\tilde{\sigma}$ formally describes a field with exponential growth in time. The overdamped modes, for which the canonically normalized field $\tilde{\sigma}$ has exponentially growing modes, correspond to non-normalizable states of the string. These modes do not have an interpretation as particle excitations of string theory.

Only when the $\sigma$ field is a tachyon, in the sense that its quadratic term in the action is

$$
-\frac{1}{2} \exp (-2 \Phi) \sqrt{-\operatorname{det} G^{(S)}} m^{2} \sigma^{2},
$$


with $m^{2}<0$, does the $\sigma$ field have an exponentially growing mode. If $\vec{k}^{2}<\left|m^{2}\right|$, we have solutions of the form

$$
\begin{aligned}
\sigma & =\exp \left(\Phi_{0}\right) \mathcal{A} \sin \vec{k} \cdot \vec{x}\left(\frac{t}{t_{0}}\right)^{\mathcal{B}_{ \pm}}, \\
\mathcal{B}_{ \pm} & =\frac{D-2}{2 q} \Gamma_{ \pm} \\
\Gamma_{ \pm} & =-q \pm \sqrt{q^{2}+\left|m^{2}\right|-\vec{k}^{2}}
\end{aligned}
$$

To be specific, the field in the first line of Eqn. (2.61), corresponding to $\mathcal{B}_{+}$, grows exponentially with time. In a generic number of dimensions, most anomaly-free string theories have tachyons of this kind. The bosonic string has a tachyon $\mathcal{T}$ whose mass $m$ satisfies $m^{2}=-\frac{4}{\alpha^{\prime}}$. In the next section we will consider particular solutions for which the bosonic string tachyon acquires an expectation value.

\section{Worldsheet description of supercritical string cos- mology}

As pointed out in the introduction, string theory in $D>D_{\text {crit }}$ is most easily studied in the timelike linear dilaton background 5 For the sake of clarity and convenience, and to introduce our analysis in the language of worldsheet conformal field theory, we briefly record a few basic and well-known facts about this theory.

The worldsheet description in conformal gauge is a two-dimensional theory of $D$ free, massless scalars $X^{\mu}$ playing the role of the embedding coordinates of spacetime. The linear dilaton enters as a modification of the definition of the two-dimensional stress tensor, relative to the $S O(D-1,1)$-invariant definition. That is, for a dilaton with gradient $\partial_{\mu} \Phi=V_{\mu}=$

\footnotetext{
${ }^{5}$ In $D \neq D_{\text {crit }}$, objects such as branes or fluxes can create a stationary point in the dilaton potential, a possibility that was studied in [27, 28. Such theories are not conveniently described by a worldsheet conformal field theory, and their $\alpha^{\prime}$ corrections are not entirely understood.
} 
const., the two-dimensional stress tensor is

$$
\begin{aligned}
& T_{++}=-\frac{1}{\alpha^{\prime}}: \partial_{\sigma^{+}} X^{\mu} \partial_{\sigma^{+}} X_{\mu}:+\partial_{\sigma^{+}}^{2}\left(V_{\mu} X^{\mu}\right) \\
& T_{--}=-\frac{1}{\alpha^{\prime}}: \partial_{\sigma^{-}} X^{\mu} \partial_{\sigma^{-}} X_{\mu}:+\partial_{\sigma^{-}}^{2}\left(V_{\mu} X^{\mu}\right)
\end{aligned}
$$

where the colons represent normal ordering of the $2 D$ theory. Here, $\sigma^{ \pm}$are particular lightcone combinations of the worldsheet coordinates $\sigma^{0,1}$ :

$$
\sigma^{ \pm}=-\sigma^{0} \pm \sigma^{1}
$$

This worldsheet theory completely defines the dynamics of a string propagating in the background of a timelike linear dilaton. Physical states of the string correspond to local operators $\mathcal{U}$ that are Virasoro primaries of weight one. That is, their operator product expansion (OPE) with the stress tensor satisfies:

$$
T_{++}(\sigma) \mathcal{U}(\tau) \simeq \frac{\mathcal{U}(\tau)}{\left(\sigma^{+}-\tau^{+}\right)^{2}}+\frac{\partial_{+} \mathcal{U}(\tau)}{\sigma^{+}-\tau^{+}}
$$

and similarly for $T_{--}$, where $\simeq$ denotes equality up to terms that are smooth as $\sigma \rightarrow \tau$.

The linear dilaton theory is free, and it exists as a CFT to all orders, and nonperturbatively, in $\alpha^{\prime}$. The theory represents a solution to the equations of motion at leading order in derivatives. Since the solution is exact, however, it must satisfy the equations of motion to all orders in derivatives. As higher derivative corrections to the string effective action are not known in closed form, the existence of such a solution constrains the form of any higher derivative corrections to the leading effective action. In this section we consider a novel set of exact solutions that deform the linear dilaton background and exhibit a nonvanishing tachyon. Following the example of the strict linear dilaton theory, we will use the existence (and exactness) of our new solutions to constrain the effective action, to leading order in derivatives and to all orders in the tachyon field $\mathcal{T}$.

\subsection{Exact solutions with nonzero tachyon}

We aim to study solutions for which the bosonic string tachyon is not everywhere equal to zero. To linearized order in the strength of the tachyon field, the deformation of the background is described in the worldsheet theory by the insertion of a single tachyon vertex 
operator into all correlation functions. The spatial component of the vertex operator is determined by the spatial dependence of the background deformation; the condition that the linearized deformation of the background satisfy the equations of motion is simply the condition that the tachyon momentum be on-shell. This, in turn, equates to the condition that the matter part of the vertex operator $\mathcal{U}_{M}$, made of the $X^{\mu}$ degrees of freedom, be a conformal primary of weight $(1,1) 6$

To be specific, a profile $\mathcal{T}(X)$ for the tachyon corresponds to the vertex operator

$$
\mathcal{U}_{M} \equiv: \mathcal{T}(X):
$$

and admits the following on-shell condition:

$$
\partial_{\mu} \partial^{\mu} \mathcal{T}(X)-2 V^{\mu} \partial_{\mu} \mathcal{T}(X)+\frac{4}{\alpha^{\prime}} \mathcal{T}(X)=0
$$

where $V_{\mu}$ is defined, per convention, as the dilaton gradient. For tachyon profiles of the form $\mathcal{T}(X)=\mu^{2} \exp \left(B_{\mu} X^{\mu}\right)$, this condition is $B^{2}-2 V \cdot B=-4 / \alpha^{\prime}$. A general value of $B_{\mu}$ will lead to a nontrivial interacting theory when the strength $\mu^{2}$ of the perturbation is treated as non-infinitesimal. For instance, the insertion of two copies of the vertex operator can lead to singularities when the positions of the vertex operators approach one another; counterterms may have to be added to preserve conformal invariance, thus complicating the analysis of the theory considerably.

There is a special set of choices for $B_{\mu}$ satisfying the equations of motion such that the $2 D$ theory is well-defined and conformal to all orders in perturbation theory, as well as nonperturbatively, in $\alpha^{\prime}$. In particular, we can choose the first term in the linearized tachyon equation of motion in Eqn. (3.5) to vanish separately, which is tantamount to choosing the vector $B_{\mu}$ to be null. It is easy to see that this renders the vertex operator : $\exp \left(B_{\mu} X^{\mu}\right)$ : non-singular in the vicinity of itself. In a free field theory, all singularities of normal-ordered operators arise from propagators contracting a free field in one operator with a free field in the second. If both operators depend only on $B \cdot X$, however, all contractions are proportional to $B_{\mu} B^{\mu}$, which vanishes when $B_{\mu}$ is null.

We therefore employ a Lorentz transformation to put $B_{\mu}$ into the form

$$
\begin{aligned}
& B_{0}=B_{1} \equiv \beta / \sqrt{2}, \\
& B_{i}=0, \quad i \geq 2 .
\end{aligned}
$$

\footnotetext{
${ }^{6}$ We will omit discussion of the ghost contribution to the vertex operator.
} 
Letting

$$
X^{ \pm} \equiv \frac{1}{\sqrt{2}}\left(X^{0} \pm X^{1}\right)
$$

the vertex operator becomes $\mathcal{U}_{M}=\mu^{2} \exp \left(\beta X^{+}\right)$. At this point we can drop the normalordering symbol because self-contractions of the null fields in the exponential are zero.

To deform the background by a finite amount, we add $\mathcal{U}_{M}$ to the worldsheet Lagrangian: this gives rise to a particularly simple quantum theory. The kinetic term for $X^{ \pm}$appears as

$$
\mathcal{L} \sim-\frac{1}{2 \pi \alpha^{\prime}}\left[\left(\partial_{\sigma^{0}} X^{+}\right)\left(\partial_{\sigma^{0}} X^{-}\right)-\left(\partial_{\sigma^{1}} X^{+}\right)\left(\partial_{\sigma^{1}} X^{-}\right)\right]
$$

The propagator for the $X^{ \pm}$fields is therefore oriented, in the sense that every propagator has an $X^{+}$at one end and an $X^{-}$at the other end. A propagator can thus be depicted by a line with an arrow pointing from $X^{+}$to $X^{-}$, as depicted in Fig. 4. The tachyon couples to

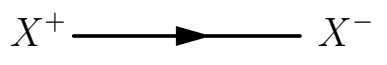

Figure 4: Oriented propagators are drawn with a directed line between $X^{+}$and $X^{-}$.

the worldsheet according to

$$
\mathcal{L} \sim-\frac{1}{2 \pi} \mu^{2} \exp \left(\beta X^{+}\right)
$$

and the equations of motion for the string appear as

$$
\begin{aligned}
\partial_{+} \partial_{-} X^{i} & =\partial_{-} \partial_{+} X^{+}=0, \quad i=2,3, \cdots, D-1 \\
\partial_{+} \partial_{-} X^{-} & =+\frac{\alpha^{\prime} \beta M^{2}}{4}
\end{aligned}
$$

where $M^{2} \equiv \mu^{2} \exp \left(\beta X^{+}\right)$.

By writing the solution to the Laplace equation for $X^{+}$as

$$
X^{+}=f_{+}\left(\sigma^{+}\right)+f_{-}\left(\sigma^{-}\right) \text {, }
$$


for arbitrary $f_{ \pm}$, the general solution for $X^{-}$can be expressed as follows:

$$
X^{-}=g_{+}\left(\sigma^{+}\right)+g_{-}\left(\sigma^{-}\right)+\frac{\alpha^{\prime} \beta \mu^{2}}{4}\left[\int_{\sigma^{+}}^{\infty} d y^{+} \exp \left(\beta f_{+}\left(y^{+}\right)\right)\right]\left[\int_{\sigma^{-}}^{\infty} d y^{-} \exp \left(\beta f_{-}\left(y^{-}\right)\right)\right]
$$

where $g_{ \pm}\left(\sigma^{ \pm}\right)$are arbitrary functions. We thus see that the theory is exactly solvable at the classical level. It is clear, however, that the classical solvability extends neatly to the quantum regime. All interaction vertices in the theory depend only on $X^{+}$, and therefore correspond to diagrams composed strictly from outgoing lines (see Fig. 51). Nontrivial Feynman diagrams,

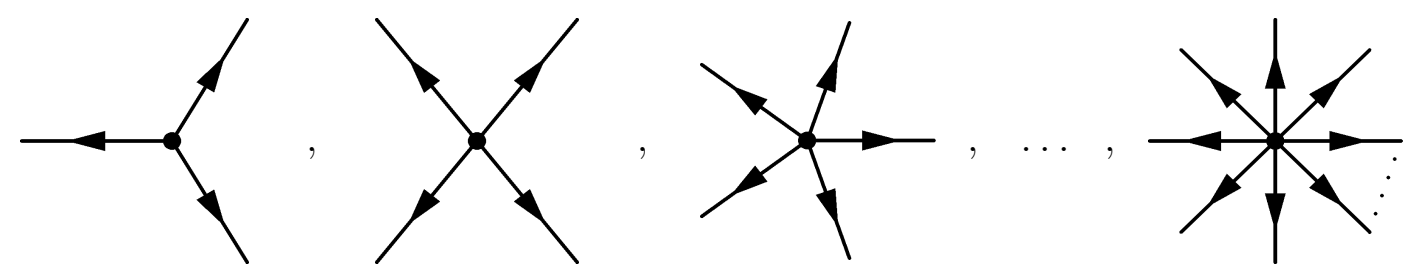

Figure 5: All interaction vertices correspond to diagrams composed of outgoing lines.

including tree graphs, must have at least two vertices connected by an internal line. Since all such diagrams are absent, we obtain a fully quantum-mechanical theory in which all amplitudes are given by their classical limits, with no quantum corrections of any kind. Note that this is only possible in a nonunitary theory: in a unitary quantum theory, sewing rules dictate nonzero values for tree and loop graphs, given a nonvanishing set of interaction vertices.7

The structure of the OPE is correspondingly simple. By virtue of the Feynman rules, all interaction contributions to OPEs are determined by those involving $X^{-}$alone. That is to say, the OPE of $X^{+}$with any other local operator is unmodified. Likewise, interaction corrections to OPEs of $X^{-}$have only operators involving $X^{+}$on the right-hand side, of the form depicted in Fig. 6. For example, we have the following relations:

\footnotetext{
${ }^{7}$ Solutions similar to the type studied here were discussed previously in various contexts [29]39]. We thank A. Tseytlin for bringing these to our attention.
} 


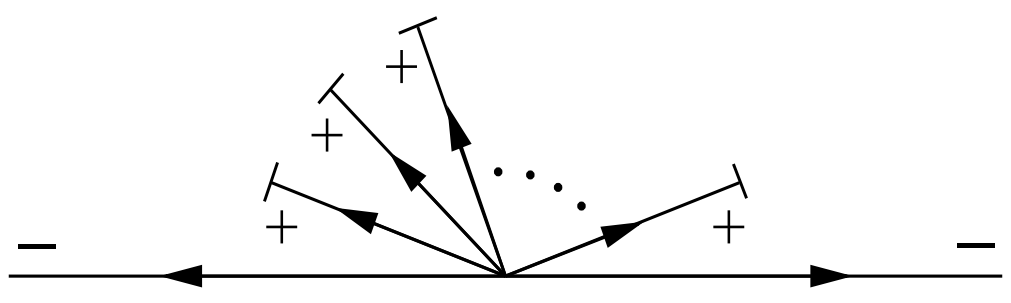

Figure 6: Generic structure of OPEs in the lightlike tachyon background.

$$
\begin{aligned}
& X^{+}(\sigma) X^{+}(\tau)=: X^{+}(\sigma) X^{+}(\tau):, \\
& X^{+}(\sigma) X^{-}(\tau)=: X^{+}(\sigma) X^{-}(\tau):+\frac{\alpha^{\prime}}{2} \ln \left|-\frac{1}{L^{2}}\left(\sigma^{+}-\tau^{+}\right)\left(\sigma^{-}-\tau^{-}\right)\right|, \\
& X^{-}(\sigma) X^{-}(\tau)=: X^{-}(\sigma) X^{-}(\tau): \\
& +\frac{\alpha^{\prime} \beta^{2}}{8} \ln \left|-\frac{1}{L^{2}}\left(\sigma^{+}-\tau^{+}\right)\left(\sigma^{-}-\tau^{-}\right)\right| \cdot \int_{\sigma^{+}}^{\tau^{+}} d y^{+} \int_{\sigma^{-}}^{\tau^{-}} d y^{-} \mu^{2} \exp \left(\beta X^{+}\right) \\
& + \text {(terms that remain smooth as } \sigma \rightarrow \tau \text { ). }
\end{aligned}
$$

We note the similarity of our theories to those of strings propagating on plane waves [40 42], in which the moduli of string theory vary along lightlike directions. The structure of Feynman diagrams, and the controlled nature of the quantum corrections are similar. In fact, the theories of [40, 41] are solvable for precisely the same reasons as the theories in this paper: the interaction vertices depend on only one of the light-cone directions, and therefore have only outgoing lines. The interaction vertices thus have no Wick contractions with themselves or each other through lines associated with the light-cone coordinates. The primary difference is that the interaction vertices in our model represent a massive (yet conformal) perturbation of the $2 D \mathrm{CFT}$, rather than a massless one.

\subsection{Physical interpretation of the solution}

The solution $\mathcal{T}(X)=\mu^{2} \exp \left(\beta X^{+}\right)$can be thought of as a phase boundary in spacetime between a $\mathcal{T} \sim 0$ phase and a $\mathcal{T}>0$ phase. The boundary is moving to the left at the speed of light. Intuitively, the $\mathcal{T}>0$ phase is a 'nothing' phase, into which no particles, including 
the graviton itself, can enter. One way to interpret this phase is to view it as an alternate description of the absence of spacetime itself.

A related spacetime-destroying solution is given by the Lorentzian continuation of the gravitational instanton of [13]. The physics of this solution can be described by first noting that the Euclidean solution exhibits time reversal symmetry at one point, say $t=0$. At that point, one matches the Euclidean instanton onto a Lorentzian solution. After that point in Lorentzian time, a bubble of nothing expands, rapidly accelerating to the speed of light at constant proper acceleration.

Viewed from the outside, the physics is very much the same as that of an ordinary Coleman-de Luccia instanton, which mediates tunneling between vacua, except that on the inside of the bubble there are no degrees of freedom at all. As a result, all matter that encounters the bubble wall is rapidly swept away and accelerated to the speed of light, along with the wall itself. It has been conjectured that the solution of [13] has an alternate description in terms of closed-string tachyon condensation, and evidence for this connection has been studied in various settings [14-22, 43, 44].

A full, classical solution for the closed string tachyon description of the bubble of nothing is not known explicitly. We propose that in the presence of a timelike linear dilaton, such a solution would approach the lightlike solution we describe in this paper at times long after the nucleation of the bubble. We should note, however, that our solution behaves differently from the bubble of nothing in flat space in one important respect: the wall in our model is not properly accelerating. This is a result of Hubble friction and the presence of the timelike linear dilaton (see Fig. 7). The drag force of the background fields stops the acceleration when the spatial thickness of the bubble is of order $\alpha^{\prime}|\dot{\Phi}| \sim \beta^{-1}$. In the absence of a dilaton gradient, the bubble wall continues to accelerate, and its spatial thickness in the frame of a given static observer blueshifts to zero as $t^{-1}$, where $t$ is the time since nucleation of the bubble.

We would also like to establish that the tachyon condensate is indeed a 'nothing' state, in which no degrees of freedom can live. This is intuitively obvious, since the high potential barrier would seem to suppress the wavefunctions of any string state that might try to penetrate the interior of the bubble. Since our theory is exactly solvable, we can do better by solving directly for the trajectory of a string colliding with the bubble wall.

Let us consider a simple example for which the string is pointlike, wherein all embedding 


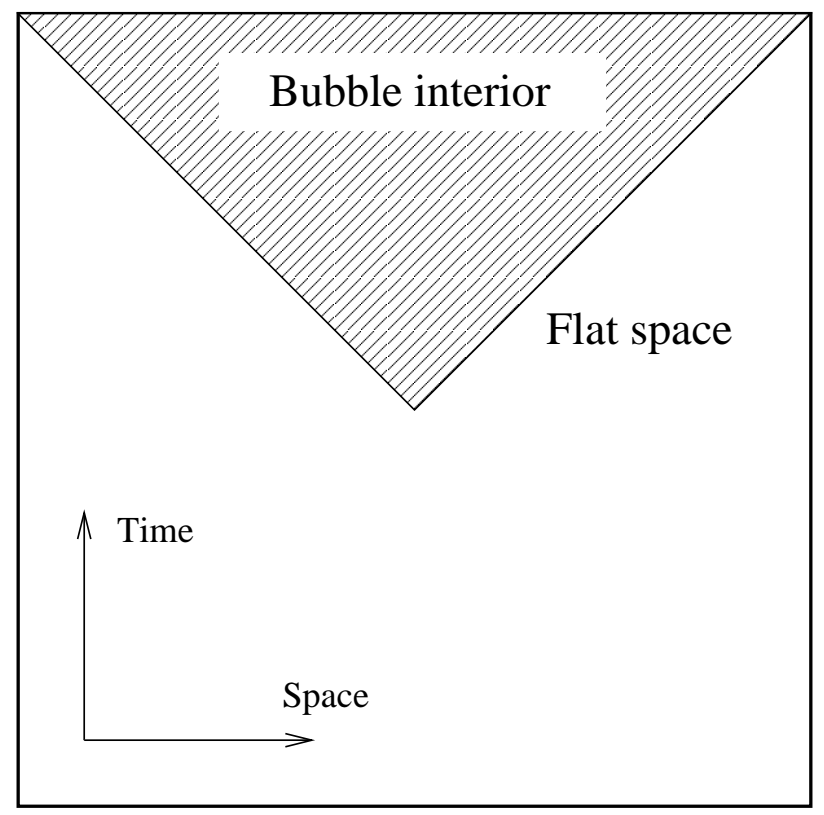

Figure 7: A bubble of nothing, which destroys spacetime. If the exterior is flat with vanishing dilaton gradient, the bubble properly accelerates all the way to future infinity. If the exterior has a flat string frame metric and timelike linear dilaton gradient, the spatial thickness of the diagonal line approaches $\alpha^{\prime}|\dot{\Phi}|$ as $t \rightarrow \infty$. Our solution corresponds to a limit that focuses on the upper left-hand corner of the diagram.

coordinates are independent of $\sigma^{1}$ and depend only on $\sigma^{0}$. The solution is then characterized by the conserved momenta $p^{i} \equiv \frac{1}{\alpha^{\prime}} \dot{X}^{i}$ and $p^{+}=\frac{1}{\alpha^{\prime}} \dot{X}^{+}$. For a pointlike solution, $X^{+}=$ $\alpha^{\prime} p^{+}\left(\sigma^{0}-\sigma_{0}^{0}\right)+X_{0}^{+}$, and $X^{-}$is given by

$$
X^{-}=\alpha^{\prime} p_{\text {initial }}^{-}\left(\sigma^{0}-\sigma_{0}^{0}\right)+\frac{\mu^{2}}{\beta \alpha^{\prime} p^{+2}} \exp \left(\alpha^{\prime} \beta p^{+}\left(\sigma^{0}-\sigma_{0}^{0}\right)\right)+X_{0}^{-},
$$

where $p_{\text {initial }}^{-}$and $X_{0}^{ \pm}$are constants of motion. Classically, the Virasoro constraint is

$$
H_{\text {worldsheet }}=-\alpha^{\prime} p^{-} p^{+}+\frac{1}{2} \alpha^{\prime} p_{i}^{2}+\mu^{2} \exp \left(\beta X^{+}\right)=0
$$

which means that

$$
p_{\text {initial }}^{-}=\frac{p_{i}^{2}}{2 p^{+}} .
$$

The physical interpretation of the solution is clear: the particle propagates at an initial 
speed

$$
v \equiv \frac{\dot{X}^{1}}{\dot{X}^{0}}=\frac{p^{+}-p_{\text {initial }}^{-}}{p^{+}+p^{-}, \text {initial }}
$$

until it hits the bubble wall, where the exponential term becomes important. At that point, the speed of the particle rapidly goes to -1 , as both the numerator and denominator are dominated by the exponential term in $p^{-}$:

$$
p^{-}=p_{\text {initial }}^{-}+\frac{\mu^{2}}{\alpha^{\prime} p^{+}} \exp \left(\alpha^{\prime} \beta p^{+}\left(\sigma^{0}-\sigma_{0}^{0}\right)\right) .
$$

The position and velocity trajectories of such a particle are plotted in Fig. 8 and Fig. 9 , respectively.

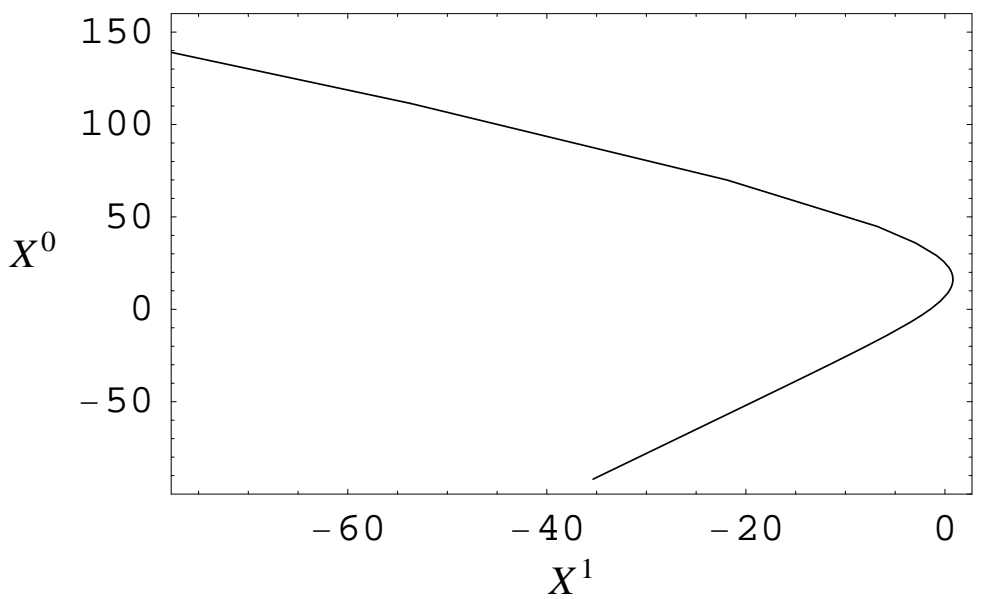

Figure 8: A plot of the position of a particle pushed by the bubble wall, as a function of time. The solution has $\mu^{2}=1, \beta=.1, X_{0}^{ \pm}=0$, and the trajectory corresponds to $p^{+}=3, H_{\perp} \equiv \frac{\alpha^{\prime} p_{i}^{2}}{2}=4$. We set $\alpha^{\prime}=1$.

\subsection{Effective actions for the dilaton-tachyon-metric system}

Effective actions for light fields in string theory, truncated to operators with low numbers of derivatives, often give a good qualitative description of some aspects of string physics. Although it is not entirely clear why the truncation to a two-derivative effective action need contain any useful information, the leading action for the metric and dilaton correctly describes many important aspects of the linear dilaton background, even quantitatively. Effective actions for this same system, augmented by tachyons, fluxes, etc., have been successfully 


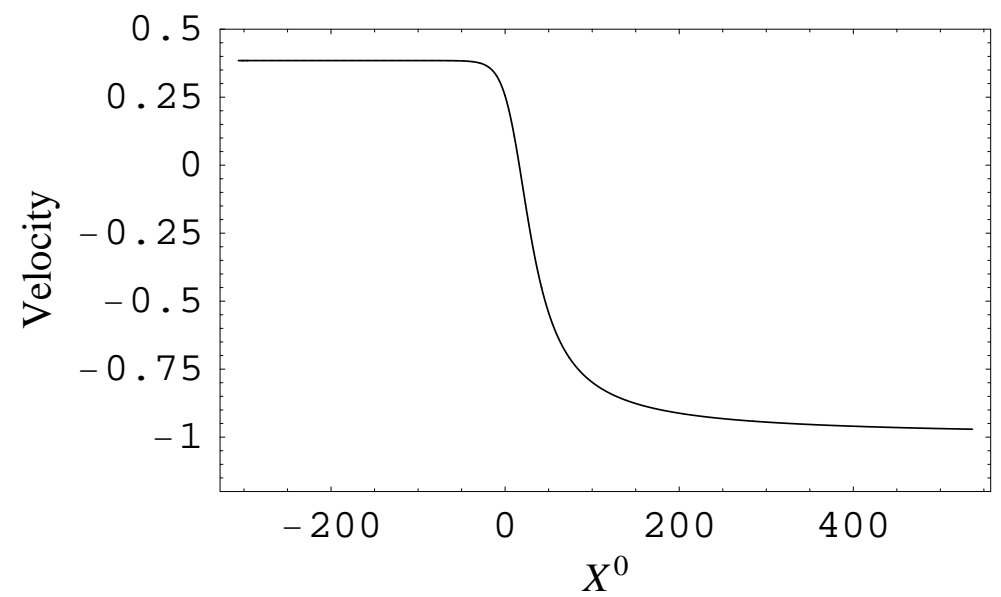

Figure 9: A plot of the velocity of a particle accelerated by the bubble wall, as a function of time. (Numerical values are set in accordance with the trajectory depicted in Fig. 8 above.)

employed to describe closed-string tachyon physics [45 56]. Furthermore, effective actions for the metric, dilaton and tachyon have been used to describe the physics of dynamical dimension change in noncritical string theories (see, e.g., [49, 51]).

Although these theories approximate the normalization of the Einstein term as a constant, independent of the tachyon condensate, it is unclear how realistic this may be. In theories of open-string tachyon condensation, the analogous dependence of the gauge field kinetic term on the tachyon must be nontrivial to correctly reproduce the qualitative physics of kink solutions and of the closed-string vacuum. We are therefore prompted to investigate constraints on the tachyon dependence of the Einstein action in a useful effective theory of closed-string tachyon condensation.

The definition of tree-level string amplitudes dictates that the dilaton dependence of the tree-level action appear as an overall factor equal to $\exp (-2 \Phi)$. The most general twoderivative ansatz for the action with this dilaton dependence is then

$$
S=\frac{1}{2 \kappa^{2}} \int d^{D} x \sqrt{\operatorname{det} G}\left[\mathcal{F}_{1} R-\mathcal{F}_{2}(\nabla \Phi)^{2}-\mathcal{F}_{3}(\nabla \mathcal{T})^{2}-\mathcal{F}_{4}-\mathcal{F}_{5} \nabla \mathcal{T} \cdot \nabla \Phi\right]
$$

where the functions $\mathcal{F}_{i}$ are defined in terms of five arbitrary functions of the tachyon as 
follows:

$$
\begin{aligned}
\mathcal{F}_{1} & \equiv e^{-2 \Phi} f_{1}(\mathcal{T}) \\
\mathcal{F}_{2} & \equiv-4 e^{-2 \Phi} f_{2}(\mathcal{T}) \\
\mathcal{F}_{3} & \equiv e^{-2 \Phi} f_{3}(\mathcal{T}) \\
\mathcal{F}_{4} & \equiv 2 e^{-2 \Phi} \mathcal{V}(\mathcal{T}) \\
\mathcal{F}_{5} & \equiv e^{-2 \Phi} f_{5}(\mathcal{T})
\end{aligned}
$$

(For convenience, we have absorbed the $\exp (-2 \Phi)$ prefactor into the $\mathcal{F}_{i}$.) The Einstein equation appears as

$$
\begin{aligned}
0= & \left(\nabla^{\mu} \nabla^{\nu}-G^{\mu \nu} \nabla^{2}+\frac{1}{2} G^{\mu \nu} G^{\rho \sigma} R_{\rho \sigma}-R^{\mu \nu}\right) \mathcal{F}_{1}-\frac{1}{2} G^{\mu \nu} \mathcal{F}_{4}+\mathcal{F}_{2} \nabla^{\mu} \Phi \nabla^{\nu} \Phi \\
& -\frac{1}{2} G^{\mu \nu} \mathcal{F}_{2}(\nabla \Phi)^{2}+\mathcal{F}_{3} \nabla^{\mu} \mathcal{T} \nabla^{\nu} \mathcal{T}-\frac{1}{2} G^{\mu \nu} \mathcal{F}_{3}(\nabla \mathcal{T})^{2}-\frac{1}{2} \mathcal{F}_{5} G^{\mu \nu} \nabla_{\rho} \mathcal{T} \nabla^{\rho} \Phi \\
& +\frac{1}{2} \mathcal{F}_{5} \nabla^{\mu} \mathcal{T} \nabla^{\nu} \Phi+\frac{1}{2} \mathcal{F}_{5} \nabla^{\nu} \mathcal{T} \nabla^{\mu} \Phi
\end{aligned}
$$

where $\nabla^{\mu}$ is the usual covariant derivative. Likewise, the dilaton and tachyon equations of motion read, respectively:

$$
\begin{aligned}
& 0=-2 R f_{1}+8 f_{2}(\nabla \Phi)^{2}-8 f_{2}^{\prime} \nabla \mathcal{T} \cdot \nabla \Phi-8 f_{2} \nabla^{2} \Phi+\left(2 f_{3}+f_{5}^{\prime}\right)(\nabla \mathcal{T})^{2}+f_{5} \nabla^{2} \mathcal{T}+4 \mathcal{V} \\
& 0=f_{1}^{\prime} R+\left(4 f_{2}^{\prime}-2 f_{5}\right)(\nabla \Phi)^{2}+f_{3}^{\prime}(\nabla \mathcal{T})^{2}-4 f_{3} \nabla \Phi \cdot \nabla \mathcal{T}-2 \mathcal{V}^{\prime}+f_{5} \nabla^{2} \Phi+2 f_{3} \nabla^{2} \mathcal{T}
\end{aligned}
$$

Following the discussion above, we assert that this theory admits a solution of the form

$$
\mathcal{T}(X)=\mu^{2} \exp \left(\beta X^{+}\right), \quad G_{\mu \nu}^{(S)}=\eta_{\mu \nu}, \quad \Phi=-q X^{0} .
$$

First, we note that Eqn. (3.5) (the on-shell condition for the tachyon profile) sets $\beta q=\frac{2 \sqrt{2}}{\alpha^{\prime}}$. This demand imposes various conditions on the functions $f_{i}(\mathcal{T})$, the simplest of which comes from the lower $(-,-)$ component of the Einstein equation (3.22):

$$
f_{2}=f_{1}
$$

With this condition in place, the $(+,-)$ component of Eqn. (3.22) (minus a multiple of the trace of (3.22) ) gives

$$
f_{5}=4 f_{1}^{\prime}
$$


and, assuming this holds, the lower $(+,+)$ component of Eqn. (3.22) yields

$$
f_{3}=-\frac{1}{\mathcal{T}} f_{1}^{\prime}-f_{1}^{\prime \prime}
$$

From this we conclude that $f_{1}^{\prime}$ must vanish at $\mathcal{T}=0$ : otherwise $f_{3}$ would be singular when the tachyon is zero. The rest of the Einstein equation, with both indices transverse to the light-cone directions, gives the condition

$$
\mathcal{V}(\mathcal{T})=\frac{D-26}{3 \alpha^{\prime}} f_{1}+\frac{4}{\alpha^{\prime}} \mathcal{T} f_{1}^{\prime}
$$

We have thus eliminated the four functions $f_{2,3,5}$ and $\mathcal{V}(\mathcal{T})$ in terms of $f_{1}$. The remaining two equations, coming from the equations of motion for the dilaton and tachyon (in Eqns. (3.23) and (3.24) above), are satisfied automatically. We therefore find that, in the presence of a nontrivial tachyon potential and nonvanishing dilaton, $f_{1}(\mathcal{T})$ must be nonconstant. In other words, the coupling of the tachyon to the Einstein term in the effective action must be nontrivial. It follows that the canonical Einstein frame metric is related to the sigma model metric via a tachyon-dependent Weyl rescaling. The Einstein frame metric of the solution described in Section 3.1 is no longer that of the linear dilaton background, or that of a homogeneous and isotropic spacetime solution of any kind. As expected, the backreaction of the tachyon on the background breaks the symmetry of the FRW satial slices.

In terms of $f_{1}$, the final effective action takes the form

$$
\begin{aligned}
S=\frac{1}{2 \kappa^{2}} \int d^{D} x \sqrt{\operatorname{det} G} e^{-2 \Phi} & {\left[f_{1} R+4 f_{1}(\nabla \Phi)^{2}+\left(\frac{1}{\mathcal{T}} f_{1}^{\prime}+f_{1}^{\prime \prime}\right)(\nabla \mathcal{T})^{2}\right.} \\
& \left.-4 f_{1}^{\prime} \nabla \mathcal{T} \cdot \nabla \Phi-\frac{2}{3 \alpha^{\prime}}(D-26) f_{1}-\frac{8}{\alpha^{\prime}} f_{1}^{\prime} \mathcal{T}\right]
\end{aligned}
$$

This form holds for all spacetime dimension $D$. We can include the critical case $D=26$ by considering a tachyon varying in the $X^{+}$direction and a dilaton varying in the $X^{-}$ direction. Doing so, we find that the constraints in Eqns. (3.26, 3.27, 3.28, 3.29) hold for the two-derivative action in the critical theory as well.

One might ask whether the equations of motion generated by our two-derivative effective action are satisfied by a background in which a tachyon condenses in a direction other than a null direction. This question is addressed in detail in reference [57].

Working to higher order in $\alpha^{\prime}$, there is an enormous freedom to choose higher-derivative terms that preserve the null tachyon background as an exact solution. The null tachyon 
solutions satisfy $\left(\partial_{\mu} \Phi\right) \cdot\left(\partial^{\mu} \mathcal{T}\right)=\frac{2}{\alpha^{\prime}} \mathcal{T}$ identically everywhere, so we can add to the action

$$
\Delta \mathcal{L} \equiv\left(\left(\partial_{\mu} \Phi\right) \cdot\left(\partial^{\mu} \mathcal{T}\right)-\frac{2}{\alpha^{\prime}} \mathcal{T}\right)^{2} \cdot \mathcal{F}\left(G_{\mu \nu}, \Phi, \mathcal{T}\right)
$$

where $\mathcal{F}$ is an arbitrary functional of the spacetime fields and their derivatives. (The modified action will still generate equations of motion that are satisfied by $\mathcal{T}=\mu^{2} \exp \left(\beta X^{+}\right), \Phi=$

$-q X^{0}, G_{\mu \nu}=\eta_{\mu \nu}$.) It would be interesting to see whether higher-derivative actions can be found that admit all known exact solutions to the equations of motion.

\section{Discussion and conclusions}

We have shown that timelike linear dilaton theories provide a simple setting for the study of time-dependent backgrounds in string theory. Quintessent theories arise naturally from string theory in supercritical dimensions $\left(D>D_{\text {crit }}\right)$. As one might expect, the equation of state $w$ of these cosmologies depends on the number of spacetime dimensions $D$. The tree level potential of supercritical string theory gives rise to the threshold value $w_{\text {crit }}=$ $-(D-3) /(D-1)$ between accelerating and non-accelerating cosmologies. For $w=w_{\text {crit }}$, the universe is globally conformally equivalent to Minkowski space. We have also described exact solutions, which behave as a bubble of nothing in these backgrounds. We have used the existence of this class of solutions to constrain the effective action of string theory.

\section{Relation to recent work}

Recently, a paper [52] appeared that examines a bubble solution related to the one presented in this paper. The authors consider a solution describing tachyon condensation along a spacelike direction (in critical spacetime dimension $D=D_{\text {crit }}$ ) with a lightlike linear dilaton:

$$
\Phi=-Q\left(X^{0}+X^{1}\right)
$$

The tachyon profile is

$$
\mathcal{T}=\mu^{2} \exp \left(-2 b X^{1}\right)
$$


with $b+\frac{1}{\alpha^{\prime} b}=Q$. They boost the solution so that the dilaton and tachyon become

$$
\begin{aligned}
\Phi & =-\frac{Q}{\gamma}\left(\tilde{X}^{0}+\tilde{X}^{1}\right) \\
\mathcal{T} & =\mu^{2} \exp \left(b \gamma\left(\tilde{X}^{0}-\tilde{X}^{1}\right)-\frac{b}{\gamma}\left(\tilde{X}^{0}+\tilde{X}^{1}\right)\right) .
\end{aligned}
$$

The authors then take a formal limit $\gamma \rightarrow \infty$ and describe the resulting theory as a simple example of a spacetime boundary in a sigma model, dropping the $\gamma^{-1}$ term in the exponent of the Liouville potential, and setting $\Phi$ to a constant. The resulting worldsheet potential is identical to our own, but only formally: the theory of [52] differs from ours at the level of observable quantities, such as operator dimensions and OPEs.

The apparent simplicity of the limit $\gamma \rightarrow \infty$ is deceptive. The most evident difficulty is that the tachyon coupling to the string worldsheet (see Eqn. (4.2)) is not marginal unless one retains the $\gamma^{-1}$ term in the Liouville exponent. Indeed, the anomalous dimension of the exponential is always equal to $-\alpha^{\prime} b^{2}$. In particular, it is of order $\gamma^{0}$. This anomalous dimension is an invariant, which cannot be boosted away.

Because the tachyon gradient appears formally null, a literal application of the approach of [52] would treat the anomalous dimension as vanishing, and the same would hold for the dilaton contribution to the dimension of the exponential (which is also of order $\gamma^{0}$ ). For a consistent treatment of the Lagrangian in [52], one needs to retain the $\gamma^{-1}$ terms in the dilaton and in the Liouville exponent. Loop corrections to the worldsheet theory are always of order unity, though some amplitudes in the theory may still be solvable by virtue of the special properties of Liouville theories.

By contrast, the tachyon exponent in our model is null from the beginning, and its anomalous dimension truly vanishes. The worldsheet theory is solvable not because of any special properties of Liouville theory, but because all loop corrections to semiclassical amplitudes vanish.

Dropping the $\gamma^{-1}$ terms for large boost also amounts to setting the terms $\partial_{\mu} \partial^{\mu} \mathcal{T},\left(\partial_{\mu} \mathcal{T}\right)^{2}$ and $\left(\partial_{\mu} \Phi\right)\left(\partial^{\mu} \mathcal{T}\right)$ to zero in the equations of motion for the spacetime fields. To analyze the constraints on the effective action consistently, one must restore them. Doing so, one recovers the constraints $(3.26,3.27,3.28)$. Additionally, if one makes an ansatz that the tachyon dependence of the Einstein term is trivial, as does [52], one finds that the tachyon potential must vanish identically for all values of $\mathcal{T}$. Allowing the Einstein term to depend 
nontrivially on the tachyon, one can accommodate a nontrivial tachyon potential, for a particular form of $f_{1}(\tilde{c})$. This result is reported in [57]. In particular, $\mathcal{V}(\mathcal{T})$ need not vanish as $\mathcal{T} \rightarrow \infty$.

\section{Outlook}

Theories describing null tachyon condensation may profitably be explored and generalized further. In particular, one can perturb the worldsheet theory of the supercritical string with an operator of the form $\exp \left(\beta X^{+}\right) \mathcal{O}$, where $\mathcal{O}$ is a relevant operator of weight $h$, and $\beta q=(1-h) 2 \sqrt{2} / \alpha^{\prime}$. The lightlike Liouville dressing of the operator still behaves classically, so we expect that such a theory should be just as solvable as the nonconformal $2 \mathrm{D}$ theory defined by perturbing with the undressed operator $\mathcal{O}$. In some cases $\mathcal{O}$ can induce a renormalization group flow to a nontrivial CFT with vanishing vacuum energy, either as a result of worldsheet supersymmetry or by fine-tuning the vacuum energy by hand. When $\mathcal{O}$ has this property, the corresponding string theory can be interpreted as a dynamical transition between two vacua with different total numbers of spacetime dimensions. We plan to address these and related issues in future studies.

\section{Acknowledgments}

We thank Nemanja Kaloper, David Kutasov, Joseph Polchinski, Savdeep Sethi, Leonard Susskind and Mark Trodden for valuable conversations. S.H. is the D. E. Shaw \& Co., L. P. Member at the Institute for Advanced Study. S.H. is also supported by U.S. Department of Energy grant DE-FG02-90ER40542. I.S. is the Marvin L. Goldberger Member at the Institute for Advanced Study, and is supported additionally by U.S. National Science Foundation grant PHY-0503584.

\section{References}

[1] A. A. Tseytlin, "On the tachyonic terms in the string effective action," Phys. Lett. B264 (1991) 311-318.

[2] A. A. Tseytlin and C. Vafa, "Elements of string cosmology," Nucl. Phys. B372 (1992) 443-466, hep-th/9109048. 
[3] A. A. Tseytlin, "Cosmological solutions with dilaton and maximally symmetric space in string theory," Int. J. Mod. Phys. D1 (1992) 223-245, hep-th/9203033.

[4] A. A. Tseytlin, "String cosmology and dilaton," hep-th/9206067.

[5] G. A. Diamandis, B. C. Georgalas, N. E. Mavromatos, E. Papantonopoulos, and I. Pappa, "Cosmological evolution in a type-0 string theory," Int. J. Mod. Phys. A17 (2002) 2241-2266, hep-th/0107124.

[6] G. A. Diamandis, B. C. Georgalas, N. E. Mavromatos, and E. Papantonopoulos, "Acceleration of the universe in type-0 non-critical strings," Int. J. Mod. Phys. A17 (2002) 4567-4589, hep-th/0203241.

[7] I. Antoniadis, C. Bachas, J. R. Ellis, and D. V. Nanopoulos, "Comments on cosmological string solutions," Phys. Lett. B257 (1991) 278-284.

[8] I. Antoniadis, C. Bachas, J. R. Ellis, and D. V. Nanopoulos, "An expanding universe in string theory," Nucl. Phys. B328 (1989) 117-139.

[9] I. Antoniadis, C. Bachas, J. R. Ellis, and D. V. Nanopoulos, "Cosmological string theories and discrete inflation," Phys. Lett. B211 (1988) 393.

[10] J. Alexandre, J. Ellis, and N. E. Mavromatos, "Non-perturbative formulation of time-dependent string solutions," JHEP 12 (2006) 071, hep-th/0610072.

[11] J. Alexandre, J. Ellis, and N. E. Mavromatos, "Non-perturbative formulation of non-critical string models," hep-th/0611228.

[12] S. Hellerman, N. Kaloper, and L. Susskind, "String theory and quintessence," JHEP 06 (2001) 003, hep-th/0104180.

[13] E. Witten, "Instability of the Kaluza-Klein vacuum," Nucl. Phys. B195 (1982) 481.

[14] G. T. Horowitz, "Tachyon condensation and black strings," JHEP 08 (2005) 091, hep-th/0506166.

[15] S. Hirano, "Energy quantisation in bulk bouncing tachyon," JHEP 07 (2005) 017, hep-th/0502199. 
[16] M. Headrick, S. Minwalla, and T. Takayanagi, "Closed string tachyon condensation: An overview," Class. Quant. Grav. 21 (2004) S1539-S1565, hep-th/0405064.

[17] M. Gutperle, "A note on perturbative and nonperturbative instabilities of twisted circles," Phys. Lett. B545 (2002) 379-383, hep-th/0207131.

[18] R. Emparan and M. Gutperle, "From p-branes to fluxbranes and back," JHEP 12 (2001) 023, hep-th/0111177.

[19] M. S. Costa and M. Gutperle, "The Kaluza-Klein Melvin solution in M-theory," JHEP 03 (2001) 027, hep-th/0012072.

[20] A. Adams, X. Liu, J. McGreevy, A. Saltman, and E. Silverstein, "Things fall apart: Topology change from winding tachyons," JHEP 10 (2005) 033, hep-th/0502021.

[21] O. Aharony, M. Fabinger, G. T. Horowitz, and E. Silverstein, "Clean time-dependent string backgrounds from bubble baths," JHEP 07 (2002) 007, hep-th/0204158.

[22] V. Fateev, A. Zamolodchikov, and A. Zamolodchikov. Unpublished.

[23] O. Aharony and E. Silverstein, "Supercritical stability, transitions and (pseudo)tachyons," hep-th/0612031.

[24] J. Polchinski, String theory. Vol. 1: An introduction to the bosonic string. University Press, Cambridge, UK, 1998.

[25] N. Seiberg, "Notes on quantum Liouville theory and quantum gravity," Prog. Theor. Phys. Suppl. 102 (1990) 319-349.

[26] A. H. Chamseddine, "A Study of noncritical strings in arbitrary dimensions," Nucl. Phys. B368 (1992) 98-120.

[27] E. Silverstein, "(A)dS backgrounds from asymmetric orientifolds," hep-th/0106209.

[28] A. Maloney, E. Silverstein, and A. Strominger, "De Sitter space in noncritical string theory," hep-th/0205316.

[29] A. A. Tseytlin, "String vacuum backgrounds with covariantly constant null Killing vector and 2-d quantum gravity," Nucl. Phys. B390 (1993) 153-172, hep-th/9209023. 
[30] A. A. Tseytlin, "Finite sigma models and exact string solutions with Minkowski signature metric," Phys. Rev. D47 (1993) 3421-3429, hep-th/9211061.

[31] J. G. Russo, L. Susskind, and L. Thorlacius, "Cosmic censorship in two-dimensional gravity," Phys. Rev. D47 (1993) 533-539, hep-th/9209012.

[32] C. G. Callan, Jr., S. B. Giddings, J. A. Harvey, and A. Strominger, "Evanescent black holes," Phys. Rev. D45 (1992) 1005-1009, hep-th/9111056.

[33] J. G. Russo and A. A. Tseytlin, "Scalar tensor quantum gravity in two-dimensions," Nucl. Phys. B382 (1992) 259-275, hep-th/9201021.

[34] H. L. Verlinde, "Black holes and strings in two dimensions," . Lectures given at Spring School on Strings and Quantum Gravity, Trieste, Italy, Apr 15-23, 1991 and at 6th Marcel Grossmann Mtg., Kyoto, Japan, Jun 23-29, 1991.

[35] T. T. Burwick and A. H. Chamseddine, "Classical and quantum considerations of two-dimensional gravity," Nucl. Phys. B384 (1992) 411-430, hep-th/9204002.

[36] A. Strominger, "Faddeev-Popov ghosts and (1+1)-dimensional black hole evaporation," Phys. Rev. D46 (1992) 4396-4401, hep-th/9205028.

[37] S. P. de Alwis, "Quantization of a theory of 2-d dilaton gravity," Phys. Lett. B289 (1992) 278-282, hep-th/9205069.

[38] A. Bilal and C. G. Callan, Jr., "Liouville models of black hole evaporation," Nucl. Phys. B394 (1993) 73-100, hep-th/9205089.

[39] S. B. Giddings and A. Strominger, "Quantum theories of dilaton gravity," Phys. Rev. D47 (1993) 2454-2460, hep-th/9207034.

[40] G. T. Horowitz and A. R. Steif, "Space-time singularities in string theory," Phys. Rev. Lett. 64 (1990) 260.

[41] G. T. Horowitz and A. R. Steif, "Strings in strong gravitational fields," Phys. Rev. D42 (1990) 1950-1959.

[42] M. Fabinger and S. Hellerman, "Stringy resolutions of null singularities," hep-th/0212223. 
[43] G. T. Horowitz and E. Silverstein, "The inside story: Quasilocal tachyons and black holes," Phys. Rev. D73 (2006) 064016, hep-th/0601032.

[44] J. McGreevy and E. Silverstein, "The tachyon at the end of the universe," JHEP 08 (2005) 090, hep-th/0506130.

[45] M. R. Douglas et al., "A new hat for the $c=1$ matrix model," hep-th/0307195.

[46] J. A. Minahan and B. Zwiebach, "Gauge fields and fermions in tachyon effective field theories," JHEP 02 (2001) 034, hep-th/0011226.

[47] J. A. Minahan and B. Zwiebach, "Effective tachyon dynamics in superstring theory," JHEP 03 (2001) 038, hep-th/0009246.

[48] J. A. Minahan and B. Zwiebach, "Field theory models for tachyon and gauge field string dynamics," JHEP 09 (2000) 029, hep-th/0008231.

[49] O. Bergman and S. S. Razamat, "Toy models for closed string tachyon solitons," hep-th/0607037.

[50] N. Moeller and H. Yang, "The nonperturbative closed string tachyon vacuum to high level," hep-th/0609208.

[51] D. Z. Freedman, M. Headrick, and A. Lawrence, "On closed string tachyon dynamics," Phys. Rev. D73 (2006) 066015, hep-th/0510126.

[52] T. Nishioka and T. Takayanagi, "AdS bubbles, entropy and closed string tachyons," hep-th/0611035.

[53] T. Suyama, "Closed string tachyons and RG flows," JHEP 10 (2002) 051, hep-th/0210054.

[54] A. Sen, "Tachyon dynamics in open string theory," Int. J. Mod. Phys. A20 (2005) 5513-5656, hep-th/0410103.

[55] A. A. Tseytlin, "Sigma model approach to string theory effective actions with tachyons," J. Math. Phys. 42 (2001) 2854-2871, hep-th/0011033. 
[56] H. Yang and B. Zwiebach, "A closed string tachyon vacuum?," JHEP 09 (2005) 054, hep-th/0506077.

[57] I. Swanson, "Cosmology of the closed string tachyon," 0804.2262. 